\title{
EFSA bewertet Bienensicherheit: Kein Verbot für Neonikotinoide gefordert - Behörde räumt zahlreiche Unsicherheitsfaktoren bei der Bewertung ein
}

Online publiziert: 1. Februar 2013

(C) Springer-Verlag Berlin Heidelberg 2013

Das Pflanzenschutzunternehmen Syngenta nahm heute zur aktuellen Bewertung von Saatgutbehandlungsmitteln, darunter auch der hauseigene Wirkstoff Thiamethoxam, durch die Europäische Sicherheitsbehörde (EFSA) Stellung.

Theo Jachmann, Geschäftsführer der Syngenta Germany GmbH sagte: „EFSA räumt ein, dass die aktuelle Bewertung mit zahlreichen Unsicherheitsfaktoren behaftet ist, denn die Richtlinie zur Bienenprüfung befindet sich noch in der Entwicklungsphase." Die Behörde beziehe sich deshalb auf die rein theoretisch vorhandenen Risiken und lasse die jahrelangen Ergebnisse von unabhängigen Monitoring-Studien aus der landwirtschaftlichen Praxis außer Acht. „Gerade die Ergebnisse aus der Praxis zeigen aber, dass die Technologie bei Beachtung der Anwendungsvorschriften sicher und ohne Probleme eingesetzt werden kann. Wir sind vor diesem Hintergrund auch nicht überrascht, dass EFSA kein Verbot der Wirkstoffgruppe fordert", so Jachmann weiter.

Bereits gestern hatte John Atkin, Mitglied im Vorstand der Syngenta International AG den Zeitdruck kritisiert, mit der die Bewertung zustande kam. Aus diesem Grunde seien bereits vorliegende wissenschaftliche und praktische Erkenntnisse überhaupt nicht berücksichtigt worden.

Seit mehr als 10 Jahren wird Thiamethoxam in mehreren europäischen Ländern zur Saatgutbehandlung eingesetzt. Die inzwischen erreichte Aussaatfläche beträgt mehrere Millionen Hektar. Bei korrekter Anwendung hat diese Technologie keinen negativen Einfluss auf die Bienengesundheit und ist deshalb in vielen Ländern der europäischen Union gängige Praxis in der landwirtschaftlichen Produktion.
Gesunde Bienenbestände sind für eine nachhaltige Nahrungsmittelerzeugung und damit indirekt auch für die gesamte Agrarbranche sehr wichtig. Es ist daher dringend notwendig, sich auf die bekannten Gefahrenquellen zu konzentrieren, die die Bienenvölker bedrohen. Dazu zählen der Befall durch die Varroa-Milbe und verschiedene Krankheitserreger sowie der Verlust von Lebensräumen und Nahrungsquellen.

Weitere Informationen zum Thema „Bienengesundheit“ finden sich auf der Internetseite www.plightofthebees.com.

Syngenta ist ein weltweit führendes Unternehmen mit mehr als 26.000 Mitarbeiterinnen und Mitarbeitern in über 90 Ländern mit einem gemeinsamen Ziel: Bringing plant potential to life. Durch erstklassige Forschung, unsere globale Präsenz und die enge Zusammenarbeit mit unseren Kunden helfen wir, die Ernteerträge und die landwirtschaftliche Produktivität zu steigern, und tragen dazu bei, die Umwelt zu schützen sowie die Gesundheit und Lebensqualität zu verbessern. Weitere Informationen unter: www. syngenta.com oder www.syngenta.de.

Pressekontakt:

Peter Hefner, Pressesprecher

Syngenta Agro GmbH

Am Technologiepark 1-5

63477 Maintal

Tel.: 06181-9081-348

E-Mail: peter.hefner@syngenta.com

Pressemeldung der Syngenta Agro GmbH 